\section{NYCOMED TRAVELGRANT}

\section{Junge Pneumologen zum ATS 2010 nach New Orleans}

Mit finanzieller Unterstützung von Nycomed Pharma konnte die Österreichische Gesellschaft für Pneumologie (ÖGP) auch 2010 den Travelgrant für junge Mitglieder der ÖGP vergeben. Mit diesem Travelgrant wurden die Kosten für den Kongress der American Thoracic Society (ATS) 2010 in New Orleans zur Gänze getragen. Bedingung war, dass die Stipendiaten Autoren eines zur Präsentation angenommenen Abstracts oder Vortrages sind.

Die ÖGP wählte aus den zahlreich eingereichten und akzeptierten Abstracts bzw. Vorträgen junger österreichischer und aufstrebender Wissenschaftler mit pneumologischem Interesse die Arbeiten von drei Autoren aus. Dr. Marie-Kathrin Breyer et al., dzt. Otto Wagner Spital, Baumgartner Höhe, 1. Interne Abteilung für Lungenkrankheiten und Ludwig Boltzmann Institut für COPD

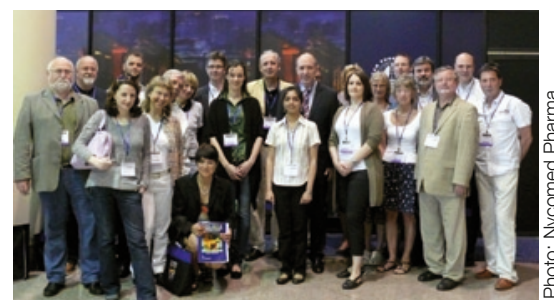

Mitarbeiter von NYCOMED Pharma, österreichische Kongressteilnehmer und die Stipendiaten in New Orleans.

mit ihrem Beitrag: „Effects of pulmonary rehabilitation on abnormal systemic levels of glucose, triglycerides, high density lipoprotein (HDL), and waist circumference in patients with COPD." Dipl. Ing. (FH) Sherin Puthenkalam et al., AKH Wien, Medizinische
Universität Wien, Univ.-Klinik für Innere Medizin II, Klinische Abteilung für Kardiologie für ihren Beitrag „,Venous thrombus resolution is accelerated by treatment with RNase“. Und Dr. Maria K. Renner et al., von derselben Abteilung für ihre Arbeit „Reinfusion of spleen cells is correcting altered thrombus organisation after splenectomy“.

Der ATS-Kongress ist einer der internationalen pneumologischen Fortbildungsveranstaltungen mit höchstem wissen-

\section{BUCHTIPP}

\section{Competitive Intelligence}

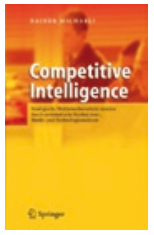

Rainer Michaeli

Competitive Intelligence Strategische Wettbewerbsvorteile erzielen durch systematische Konkurrenz-, Markt- und tische Konkurrenz-,
Technologieanalysen Technologieanalysen
629 Seiten, Springer, Berlin 2006, ISBN: 978-3-540-0308-1-2, Preis: EUR 79,95

Zur Entwicklung einer erfolgreichen Unternehmensstrategie, einer vorausschauenden Planung und der Erreichung eines Wettbewerbsvorteils bedarf es der strategischen Wettbewerbsanalyse. Dies gilt im privatwirtschaftlichen Bereich aber auch in der Profilierung der einzelnen öffentlich rechtlichen Organisationen. Krankenhäuser haben im finanziell hart umkämpften Gesundheitsmarkt in Wahrheit keine Ausnahmestellung. Das Konzept der „Competitive Intelligence" hilft dabei die Einzelinformationen über Markt und Wettbewerb durch gezielte Erhebung und Analyse zu einem sinnvollen Ganzen zusammenzusetzen und damit wirkungsvolle Zukunftsstrategien zu entwerfen und auch umsetzen zu können. Intelligence steht hier für Informationen, die erhoben werden, und stammt aus dem militärischen Bereich, wo er die umfassendere Bedeutung von schaftlichem Niveau. Neben den Arbeiten über Grundlagenforschung werden in den Posterpräsentationen auch die neuesten Ergebnisse über therapeutische Möglichkeiten und klinische Erfahrung präsentiert. Dabei ist ein intensiver persönlicher Wissensaustausch und Kontakt gegeben, der für die weitere Arbeit einen wesentlichen Stimulus darstellt.

Quelle: Presseaussendung Nycomed Pharma

Aufklärung darstellte. Rainer Michaeli ist Geschäftsführer der auf Technologie- und Wirtschaftsberatung spezialisierten Gesellschaft DIE DENKFABRIK und Direktor des Instituts für Competitive Intelligence in Deutschland. Er kennt die Praxis und die theoretischen Grundlagen und er vermittelt in Seminaren und Schulungen diese Fähigkeiten zur Gewinnung und Analyse der Informationen an die Praktiker. Seine theoretischen und praktischen Erfahrungen hat Michaeli nun unter Mitarbeit von Koautoren in einem umfassenden Buch zusammengefasst und bietet darin „Lösungsansätze, Ideen und Techniken, um effektive Recherchen zu führen, die Informationsflut zu beherrschen, Analysetechniken effizient zu nutzen, $\mathrm{Cl}$ erfolgreich als Prozess in einem Unternehmen zu implementieren und strategische Entscheidungen mit größerer Sicherheit zu treffen."

Die Möglichkeiten, Informationen zu erheben sind vielfältig. Sie können über die veröffentlichten Informationen in Print- und elektronischen Medien ebenso erfolgen, wie über Umfragen oder Datenbanken. Die Kunst ist, die richtigen Informationen zu finden und auch zu nutzen. Dazu leistet dieses Handbuch mit theoretischem Unterbau und vielen Praxisbeispielen einen grundlegenden Beitrag.

\title{
IMPRESSUM
}

Herausgeber und Verleger: Springer-Verlag GmbH, Professional Media, Sachsenplatz 4-6, P.O.Box 89, 1201 Wien, Austria, Tel.: 01/330 24 15, Fax: 01/330 24 26-260, Internet: www.springer.at, www.SpringerMedizin.at; Geschäftsführung: Mag. Katharina Oppitz; Leitung: Mag. Margarete Zupan; Redaktion: Verantwortlicher Redakteur: Dr. Verena Kienast; Mitarbeiter dieser Ausgabe: Dr. Renate Höhl, Mag. Patricia Herzberger, Mag. Tanja Fabsits; Redaktionssekretariat: Susanna Hinterberger; Portalmanagement SpringerMedizin.at: Andrea Niemann; Produktion: Mag. (FH) Dorothea Woliński; Anzeigen: Dipl. Tzt. Elise Haidenthaller; Es gilt die Anzeigenpreisliste 2010; Erscheinungsweise: 6x jährlich; Abonnement: Michaela Bolli; Wiener klinisches Magazin ist eine Beilage zur Wiener klinischen Wochenschrift (WKW); Bezugspreis pro Jahr: EUR 43,-- zuzüglich MwSt. und Versandkosten; Verlagsort: Wien; Herstellungsort: Wien; Erscheinungsort: Wien; Verlagspostamt: 1210 Wien P.b.b.; ISSN Print: 1869-1757; ISSN Electronic: 1613-7817; Band 13, Heft 6/2010; Inhalte des „Wiener Klinischen Magazins“ sind ab Dezember 2010 auch über die Zeitungsdatenbank der APA (http://www.defacto.at) abrufbar. Layout: Ursula Szorger; Design: Wojtek Grzymala; Druck: Holzhausen Druck \& Medien GmbH, 1140 Wien-Auhof, Holzhausenplatz 1.

Alle namentlich gekennzeichneten Beiträge spiegeln nicht unbedingt die Meinung der Redaktion wider. Diese Beiträge fallen somit in den persönlichen Verantwortungsbereich des Verfassers. Die Redaktion übernimmt keine Haftung für unaufgefordert eingesandte Manuskripte. Mit „Sonderbericht“ oder „Advertorial“ gekennzeichnete Seiten sind entgeltliche Einschaltungen nach \$26 Mediengesetz. Allgemeiner Teil/Rechtliche Hinweise für Autoren: Die Autorin/der Autor erklärt, dass ihr/sein Manuskript in dieser Form bislang nicht anderweitig veröffentlicht oder zur Veröffentlichung eingereicht wurde. Die Autorin/der Autor überträgt mit der Übergabe des fertigen Manuskripts und der Veröffentlichung in der Fachzeitschrift die notwendigen Nutzungsrechte zur Vervielfältigung und Verbreitung an den Verlag, insbesondere das Recht der Nutzung zu gewerblichen Zwecken durch Druck, Nachdruck, Verbreitung in elektronischer Form oder andere Verfahren und Medien durch Springer Science + Business Media. Die Autorin/der Autor holt, falls notwendig, die Nutzungsrechte an Texten und Bildern Dritter vor Übergabe des fertigen Manuskripts ein, eventuelle Ansprüche Dritter sind somit geklärt. Hinweise zur Verwertung: Die Zeitschrift sowie alle in ihr enthaltenen einzelnen Beiträge und Abbildungen sind urheberrechtlich geschützt. Jede Verwertung, auch auszugsweise, die nicht ausdrücklich vom Urheberrechtsgesetz zugelassen ist, bedarf der vorherigen schriftlichen Zustimmung des Verlages. Das gilt insbesondere für Vervielfältigungen, Bearbeitungen, Übersetzungen, Mikroverfilmungen und die Verarbeitung in elektronischen Systemen. Produkthaftung: Die Wiedergabe von Gebrauchsnamen, Handelsnamen, Warenbezeichnungen usw. in dieser Zeitschrift berechtigt auch ohne besondere Kennzeichnung nicht zu der Annahme, dass solche Namen im Sinne der Warenzeichen- und Markenschutz-Gesetzgebung als frei zu betrachten wären und daher von jedermann benutzt werden dürften. Angaben über Dosierungsanweisungen und Applikationsformen sind anhand anderer daher von jedermann benutzt werden dürften. Angaben über Dosierungsanweisungen und Applikationsformen sind an
Literaturstellen oder der Packungsbeilage auf ihre Richtigkeit zu überprüfen. Der Verlag übernimmt hierfür keine Gewähr. Eigentümer und Copyright-Inhaber: @ 2010 Springer-Verlag/Wien. SpringerWienNewYork ist ein Unternehmen von Springer Science+Business Media; Beilage zur Wiener klinischen Wochenschrift 23-24/2010 